\title{
An analytic description of the radio emission of air showers based on its emission mechanisms
}

\author{
Christian Glaser ${ }^{1,2, \star}$, Sijbrand de Jong ${ }^{3,4}$, Martin Erdmann ${ }^{1}$, and Jörg R. Hörandel $\left.\right|^{3,4}$ \\ ${ }^{1}$ RWTH Aachen University, III. Physikalisches Institut A, Aachen, Germany \\ ${ }^{2}$ Department of Physics and Astronomy, University of California, Irvine, USA \\ ${ }^{3}$ IMAPP, Radboud University Nijmegen, Nijmegen, Netherlands \\ ${ }^{4}$ Nikhef, Science Park, Amsterdam, Netherlands
}

\begin{abstract}
The spatial signal distribution of the radio frequency radiation from extensive air showers on the ground contains information on crucial cosmic-ray properties, such as energy and mass. A long-standing challenge to access this information experimentally with a sparse grid of antennas is an analytic modeling of the radio signal distribution, which will be addressed in this contribution. We present an analytic model based on the two physical processes generating radio emission in air showers: the geomagnetic and the charge-excess emission. Our study is based on full Monte-Carlo simulations with the CoREAS code. Besides an improved theoretical understanding of radio emission, our model describes the radio signal distribution with unprecedented precision. Our model explicitly includes polarization information, which basically doubles the information that is used from a single radio station. The model depends only on the definition of the shower axis and on the parameters energy and distance to the emission region, where the distance to the emission region has a direct relation to the cosmic-ray mass. The model describes the true signal distribution precisely such that the model uncertainties are negligible compared to typical experimental uncertainties.
\end{abstract}

\section{Introduction}

The radio technique measures air showers with a duty cycle close to $100 \%$ and allows for a precise and accurate determination of the cosmic-ray energy and mass [1-4]. The determination of the cosmic-ray energy is competitive to existing techniques in terms of achievable accuracy [5,6] given a thorough detector calibration [7]. The main advantages are that the atmosphere is transparent to radio waves and that the radiation originates only from the well-understood electromagnetic part of the air shower which allows for a precise theoretical calculation of the radio emission [8].

Both cosmic-ray properties can be extracted from the spatial signal distribution: The cosmic-ray energy is directly related to the radiation energy, i.e., the energy emitted by the air shower in form of radio waves, which can be measured experimentally by integrating over the spatial signal distribution. The cosmic-ray mass is estimated via the distance to the shower maximum $D_{X_{\max }}$ which determines the shape of the spatial signal distribution. To access this information experimentally from a sparse grid of antennas, an analytic modeling of the signal distribution is required.

^e-mail: christian.glaser@uci.edu, CG is supported by the German Research Foundation, (DFG), on grant GL 914/1-1. 

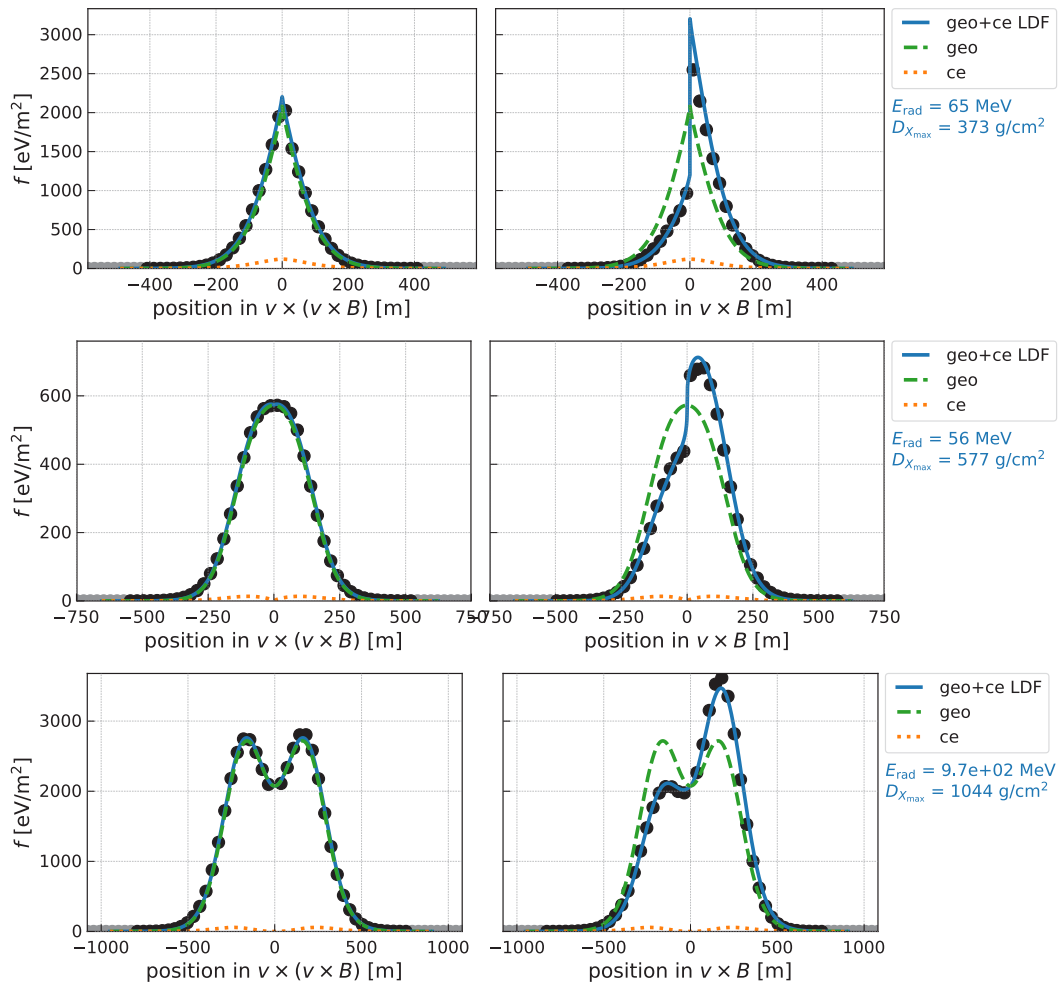

Figure 1. The energy fluence of an air shower on the ground is shown along the $\vec{v} \times(\vec{v} \times \vec{B})$ axis (no interference between geomagnetic and charge-excess) and along the $\vec{v} \times \vec{B}$ axis (maximum interference between geomagnetic and charge-excess) for $D_{X_{\max }}=374 \mathrm{~g} / \mathrm{cm}^{2}$ (top), $D_{X_{\max }}=572 \mathrm{~g} / \mathrm{cm}^{2}$ (middle) and $D_{X_{\max }}=1046 \mathrm{~g} / \mathrm{cm}^{2}$ (bottom). Figure from [12].

We developed a new analytic model that depends only on the radiation energy, the distance to the emission region $\left(D_{X_{\max }}\right)$ and the shower axis. Thus, the parameters of our model directly relate to the cosmic-ray properties energy and mass. This is achieved by modeling the signal originating from the geomagnetic and charge-excess emission separately, and building on the improved understanding obtained during the recent years [9-11]. In the following we briefly describe our model and discuss its precision. A detailed documentation can be found in [12] and a reference implementation is available at [13].

\section{Shapes of the signal distribution}

We study the spatial distribution of the radio signal with an extensive set of CoREAS [14] simulations. We find that the shape of the signal changes with increasing $D_{X_{\max }}$ from a peaked distribution to a Gaussian like distribution to a double peak distribution. We present typical examples of these shapes in Fig. 1. The left-right asymmetry along the $\vec{v} \times \vec{B}$ axis is due to the interference between the geomagnetic and charge-excess emission. The geomagnetic and charge-excess functions itself are radially symmetric and can be modeled with the following functions: 


$$
f_{\mathrm{geo}}=\left\{\begin{array}{ll}
\frac{1}{N_{R_{-}}} E_{\mathrm{geo}} \exp \left(-\left(\frac{r-R_{\mathrm{geo}}}{\sqrt{2} \sigma_{\mathrm{geo}}}\right)^{2}\right. & \text { if } R_{\mathrm{geo}}<0 \\
\frac{1}{N_{R_{+}}} E_{\mathrm{geo}}\left[\exp \left(-\left(\frac{r-R_{\mathrm{geo}}}{\sqrt{2} \sigma_{\mathrm{geo}}}\right)^{2}\right)+\exp \left(-\left(\frac{r+R_{\mathrm{geo}}}{\sqrt{2} \sigma_{\mathrm{geo}}}\right)^{2}\right)\right] & \text { if } R_{\mathrm{geo}} \geq 0
\end{array},\right.
$$

and

$$
f_{\mathrm{ce}}(r)=\frac{1}{N_{\mathrm{ce}}} E_{\mathrm{ce}} r^{k} \exp \left(\frac{-r^{2}(k+1)}{2 \sigma_{\mathrm{ce}}^{2}}\right) .
$$

The functions are normalized such that the parameters $E_{\mathrm{geo}}$ and $E_{\mathrm{ce}}$ are the radiation energies of the geomagnetic and charge-excess emission. The parameter $R_{\text {geo }}$ can be interpreted as the radius of the Cherenkov ring. The parameters $\sigma_{\text {geo }}$ and $\sigma_{\text {ce }}$ describe the width of the function and the parameter $k$ is an additional shape parameter of the charge-excess function. All shape parameters correlate with $D_{X_{\max }}$ and can be parametrized accordingly. Furthermore, we can express $E_{\mathrm{geo}}$ and $E_{\mathrm{ce}}$ as a function of the total radiation energy and the air density at the emission (that can be calculated from $D_{X_{\max }}$ ) using the result of [11]. Hence, we can formulate our model in a way that it depends only on the physical quantities radiation energy and $D_{X_{\max }}$.

\section{Precision and application of the analytic model}

We quantify the precision of the analytic model by reconstructing the radiation energy and $D_{X_{\max }}$ from the simulated spatial distribution of the radio signal using our model. We find an excellent resolution of the radiation energy of $4 \%$ and a $D_{X_{\max }}$ resolution of $13 \mathrm{~g} / \mathrm{cm}^{2}$ which is much smaller than the typical experimental uncertainties of sparse radio arrays. Hence, our model is particularly useful to precisely determine the cosmic-ray energy and mass from radio air-shower measurements, and has already been successfully used by the Pierre Auger Observatory [15] and the LOFAR cosmic-ray detector [16].

\section{References}

[1] A. Aab et al., Phys. Rev. Lett. 116 (24) (2016) 241101

[2] A. Aab et al., Phys. Rev. D 93 (12) (2016) 122005

[3] S. Buitink et al., Nature 531 (7592) (2016) 70-73

[4] P.A. Bezyazeekov et al. - Tunka-Rex Collaboration, JCAP 01(2016)052

[5] C. Glaser, Ph.D. thesis, RWTH Aachen University (2017)

[6] R. Krause for the Pierre Auger Collaboration, PoS(ICRC2017)528

[7] A. Aab et al., 2017 JINST 12 T10005

[8] M. Gottowik, C. Glaser, T. Huege, J. Rautenberg, Astroparticle Physics 103 (2018) 87-93

[9] J. Alvarez-Muñiz, W. R. Carvalho, H. Schoorlemmer, E. Zas, Astropart. Phys. 59 (2014) 29-38

[10] A. Nelles et al., Astropart. Phys. 60 (2015) 13-24

[11] C. Glaser, M. Erdmann, J. R. Hörandel, T. Huege, J. Schulz, JCAP 09(2016)24

[12] C. Glaser, S. de Jong, M. Erdmann, J. Hörandel, Astroparticle Physics 104 (2019) 64-77

[13] GeoCELDF Reference Implementation. https://github.com/cg-laser/geoceLDF

[14] T. Huege, M. Ludwig, C. W. James, AIP Conf. Proc. 1535 (2013) 128-132

[15] F. Canfora for the Pierre Auger Collaboration, Proc. ARENA2018, Catania, Italy June 2018

[16] I. Plaisier et al., Proc. ARENA2018, Catania, Italy June 2018 